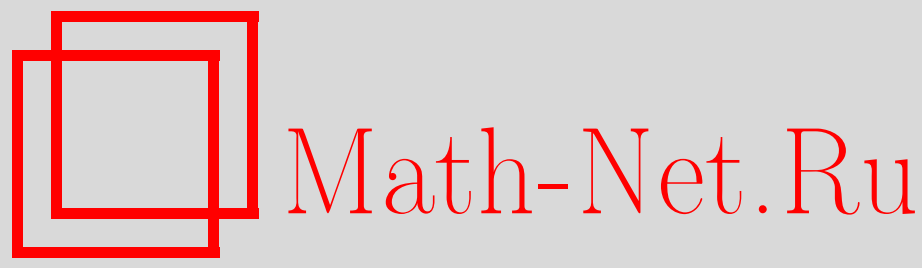

У. А. Розиков, О. Н. Хакимов, $p$-Адические меры Гиббса и марковские случайные поля на счетных графах, $T M \Phi$, 2013, том 175, номер 1, 84-92

DOI: https://doi.org/10.4213/tmf8428

Использование Общероссийского математического портала Math-Net.Ru подразумевает, что вы прочитали и согласны с пользовательским соглашением http://www.mathnet.ru/rus/agreement

Параметры загрузки:

IP : 54.198 .187 .58

26 апреля 2023 г., 15:06:19

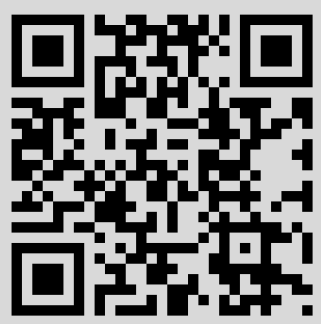




\title{
p-АДИЧЕСКИЕ МЕРЫ ГИББСА И МАРКОВСКИЕ СЛУЧАЙНЫЕ ПОЛЯ НА СЧЕТНЫХ ГРАФАХ
}

\begin{abstract}
Известно, что в вещественнозначном случае понятия меры Гиббса и марковского случайного поля совпадают. Однако в $p$-адическом случае класс $p$-адических марковских случайных полей шире, чем класс $p$-адических мер Гиббса. Построены $p$-адические марковские случайные поля (на конечных графах), которые не являются $p$-адическими мерами Гиббса. Дано определение $p$-адического марковского случайного поля на счетных графах. Показано, что множество таких полей является непустым замкнутым подпространством на множестве всех р-адических вероятностных мер.
\end{abstract}

Ключевые слова: граф, конфигурация, p-адическая мера Гиббса, p-адические марковские случайные поля.

DOI: $10.4213 / \operatorname{tmf} 8428$

\section{1. ВВЕДЕНИЕ}

Вещественнозначные меры Гиббса возникают во многих проблемах теории вероятностей и статистической механики. Эта мера, связанная с распределением Больцмана, обобщает понятие канонического ансамбля. Кроме того, мера Гиббса - уникальная мера, которая максимизирует энтропию для данной ожидаемой энергии; таким образом, мера Гиббса лежит в основе максимальных методов энтропии и соответствующих алгоритмов. Теория меры Гиббса хорошо развита (см. [1]-[3]), но неархимедов ( $p$-адический) аналог меры Гиббса изучен мало.

Известно [4]-[6], что $p$-адические модели в физике не могут быть описаны с использованием обычной теории вероятностей. В работе [7] абстрактная $p$-адическая теория вероятностей была развита посредством теории неархимедовых мер [8]. Вероятностные процессы на поле $p$-адических чисел были изучены многими авторами (см., например, [9]-[11]). В этих работах широкие классы процессов Маркова были построены и изучены на поле $p$-адических чисел.

В нашем случае вероятностные меры имеют значения в поле $p$-адических чисел. Это приводит к некоторым трудностям. Например, нет никакой информации

${ }^{*}$ Институт математики при Национальном университете Узбекистана им. М. Улугбека, Ташкент, Узбекистан. E-mail: rozikovu@yandex.ru, hakimovo@mail.ru 
о компактности множества p-адических мер. Неархимедов аналог теоремы Колмогорова был доказан в работе [12]. Такой результат позволяет строить широкие классы $p$-адических вероятностных мер, используя конечномерные распределения вероятности. Поэтому есть возможность развивать $p$-адическую статистическую механику.

В настоящей работе мы изучаем $p$-адические марковские случайные поля и $p$-адические меры Гиббса на не более чем счетных графах. Структура работы следующая. В разделе 2 даются основные определения и известные факты. Раздел 3 посвящен случаю конечного графа. В разделе 4 мы строим $p$-адические марковские случайные поля, которые не являются $p$-адическими мерами Гиббса. В разделе 5 исследуются счетные графы, и мы даем определение $p$-адического марковского случайного поля на счетных графах. Показано, что множество таких полей является непустым замкнутым подпространством в множестве всех $p$-адических вероятностных мер.

Конкретные примеры $p$-адических мер Гиббса для моделей статистической физики были изучены в работах [13]-[16].

\section{2. ОПРЕДЕЛЕНИЯ И ФАКТЫ}

Каждое рациональное число $x \neq 0$ может быть представлено в виде $x=p^{r}(n / m)$, где $r, n \in \mathbb{Z}, m$ - положительное число, $(n, m)=1$, причем $m$ и $n$ не делятся на $p$, а $p-$ фиксированное простое число. $p$-Адическая норма $|x|_{p}$ определяется по формуле

$$
|x|_{p}= \begin{cases}p^{-r}, & x \neq 0 \\ 0, & x=0 .\end{cases}
$$

Эта норма удовлетворяет сильному неравенству треугольника:

$$
|x+y|_{p} \leqslant \max \left\{|x|_{p},|y|_{p}\right\} .
$$

Это свойство показывает неархимедовость нормы. Из него непосредственно следуют следующие свойства:

1) если $|x|_{p} \neq|y|_{p}$, то $|x-y|_{p}=\max \left\{|x|_{p},|y|_{p}\right\}$;

2) если $|x|_{p}=|y|_{p}$, то $|x-y|_{p} \leqslant|x|_{p}$.

Пополнение поля рациональных чисел $\mathbb{Q}$ по $p$-адической норме приводит к полю $p$-адических чисел $\mathbb{Q}_{p}$ для каждого простого $p[17]$.

Начав с поля рациональных чисел $\mathbb{Q}$, мы можем получить либо поле вещественных чисел $\mathbb{R}$, либо одно из полей $p$-адических чисел $\mathbb{Q}_{p}$ (теорема Островского).

Для $a \in \mathbb{Q}_{p}$ и $r>0$ обозначим

$$
B(a, r)=\left\{x \in \mathbb{Q}_{p}:|x-a|_{p}<r\right\} .
$$

p-Адический логарифм определяется как ряд:

$$
\log _{p}(x)=\log _{p}(1+(x-1))=\sum_{n=1}^{\infty}(-1)^{n+1} \frac{(x-1)^{n}}{n},
$$

он сходится для $x \in B(1,1) ; p$-адическая экспонента определяется как

$$
\exp _{p}(x)=\sum_{n=0}^{\infty} \frac{x^{n}}{n !}
$$


этот ряд сходится для $x \in B\left(0, p^{-1 /(p-1)}\right)$.

Лемма 1 [17]. Пусть $x \in B\left(0, p^{-1 /(p-1)}\right)$. Тогда

$$
\begin{gathered}
\left|\exp _{p}(x)\right|_{p}=1, \quad\left|\exp _{p}(x)-1\right|_{p}=|x|_{p}, \quad\left|\log _{p}(1+x)\right|_{p}=|x|_{p} \\
\log _{p}\left(\exp _{p}(x)\right)=x, \quad \exp _{p}\left(\log _{p}(1+x)\right)=1+x
\end{gathered}
$$

\section{3. СЛУЧАЙ КОНЕЧНЫХ ГРАФОВ}

Пусть $\Lambda$ - конечное множество, а точки множества $\Lambda$ являются вершинами некоторого конечного графа $\Gamma=(\Lambda, e)$, где $e-$ множество ребер графа $\Gamma$. Через $P(\Lambda)$ обозначим множество всех подмножеств в $\Lambda$. Будем предполагать, что $\Gamma$ не имеет кратных ребер или петель, и используем следующие определения и обозначения. Если $x, y \in \Lambda$ и существует ребро графа, соединяющее $x$ и $y$, то $x$ и $y$ называются соседями. Пусть отображение $c: \Lambda \times \Lambda \rightarrow\{0,1\}$ определено формулой

$$
c(x, y)= \begin{cases}1, & x \text { и } y-\text { соседи } \\ 0 & \text { в противном случае. }\end{cases}
$$

Заметим, что $c(x, x)=0$ для всех $x \in \Lambda$, поскольку мы предположили, что $\Gamma$ не имеет петель. Если $A \subset \Lambda$, то граница $A$ есть множество $\partial A \in P(\Lambda)$, определяемое равенством $\partial A=\{y \in \Lambda \backslash A: c(x, y)=1$ для некоторого $x \in \Lambda\}$. Для $x \in \Lambda$ мы будем писать $\partial x$ вместо $\partial\{x\}$ (и вообе будем стремиться по возможности писать $x$ вместо $\{x\}$; например, если $A \in P(\Lambda)$, то запись $A \cup x$ предпочтительнее, чем $A \cup\{x\})$. Для любого $A \in P(\Lambda)$ через $|A|$ обозначим число точек в $A$.

Пусть $\Phi=\{1,2, \ldots, q\}$. Функция $\sigma: \Lambda \rightarrow \Phi$ называется конфигурацией. Обозначим через $\Omega$ множество всех конфигураций на $\Lambda$. Заметим, что при $q=2$ множество $\Omega$ можно отождествить с множеством всех подмножеств множества $\Lambda$, т. е. $\Omega=P(\Lambda)$. Для $A \subset \Lambda$ через $\sigma_{A}$ обозначим сужения конфигурации $\sigma$ на $A$, а через $\Omega_{A}-$ множество всех конфигураций на $A$.

Пусть $\mathcal{M}_{p}(\Omega)$ обозначает множество всех $p$-адических вероятностных мер на $(\Omega, \Sigma)$ (при условии, что на $\Omega$ задана алгебра $\Sigma$ ), т. е. измеримыми являются все подмножества из $\Sigma$. Элемент $\mu \in \mathcal{M}_{p}(\Omega)$ обозначает $p$-адическое распределение конфигураций, когда система находится в состоянии динамического равновесия. Поэтому мы будем называть $\mathcal{M}_{p}(\Omega)$ множеством $p$-адических состояний на $(\Omega, \Sigma)$. Поскольку $\Omega$ - конечное множество, мы отождествим вероятности, т. е. мы рассматриваем $\mu$ как функцию из $\Omega$ в $\mathbb{Q}_{p}$, обладающую следующими свойствами:

1) $\mu(\sigma) \neq 0$ для всех $\sigma \in \Omega$;

2) $\sum_{\sigma \in \Omega} \mu(\sigma)=1$.

3.1. $p$-Адическое марковское случайное поле. Пусть множества $A, B \subset \Lambda$, причем $A \cap B=\varnothing$. Обозначим через

$$
\mu\left(\bar{\sigma}_{A} \mid \tilde{\sigma}_{B}\right)=\frac{\sum_{\sigma_{\Lambda \backslash(A \cup B)}} \mu\left(\bar{\sigma}_{A}, \tilde{\sigma}_{B}, \sigma_{\Lambda \backslash(A \cup B)}\right)}{\sum_{\sigma_{\Lambda \backslash(A \cup B), \bar{\sigma}_{A}}} \mu\left(\bar{\sigma}_{A}, \widetilde{\sigma}_{B}, \sigma_{\Lambda \backslash(A \cup B)}\right)}
$$

условную вероятность того, что конфигурация $\sigma$ есть $\bar{\sigma}_{A}=\{\bar{\sigma}(x), x \in A\}$ на множестве $A$ при условии, что на множестве $B$ она равна $\widetilde{\sigma}_{B}=\{\widetilde{\sigma}(x), x \in B\}$. 
ОПРЕДЕЛЕНИЕ 1. $p$-Адическая вероятностная мера $\mu \in \mathcal{M}_{p}(\Omega)$ называется $p$ - $a \partial u$ ческим марковским случайным полем, если

$$
\mu\left(\bar{\sigma}_{A} \mid \tilde{\sigma}_{B}\right)=\mu\left(\bar{\sigma}_{A} \mid \tilde{\sigma}_{\partial A \cap B}\right) \quad \text { для всех } A, B \subset \Lambda \text {. }
$$

Это означает, что вероятность того, что конфигурация $\sigma$ есть $\bar{\sigma}_{A}=\{\bar{\sigma}(x), x \in A\}$ на множестве $A$ при условии, что на множестве $B$ она равна $\widetilde{\sigma}_{B}=\{\tilde{\sigma}(x), x \in B\}$, совпадает с вероятностью того, что конфигурация $\sigma$ равна $\bar{\sigma}_{A}=\{\bar{\sigma}(x), x \in A\}$ на множестве $A$ при условии, что на множестве $\partial A \cap B$ она равна $\widetilde{\sigma}_{\partial A \cap B}=\{\widetilde{\sigma}(x)$, $x \in \partial A \cap B\}$.

3.2. $p$-Адическое гиббсовское состояние. Рассмотрим состояние на $(\Omega, \Sigma)$, являющееся $p$-адическим гиббсовским состоянием. Функция $H: \Omega \rightarrow \mathbb{Q}_{p}$ называется гамильтонианом на $\Omega$. Для любого гамильтониана $H$ на $\Omega$ с условием $|H(\sigma)|_{p}<$ $p^{-1 /(p-1)}$ мы определим $p$-адическое гиббсовское состояние с гамильтонианом $H$ как состояние $\pi \in \mathcal{M}_{p}(\Omega)$ :

$$
\pi(\sigma)=Z^{-1} \exp _{p}\{-H(\sigma)\}
$$

где $Z$ - нормирующая константа, т. е.

$$
Z=\sum_{\sigma \in \Omega} \exp _{p}\{-H(\sigma)\}
$$

Пусть $H$ - гамильтониан на $\Omega, A$ - подмножество из $\Lambda$. Через $H_{A}\left(\sigma_{A}\right)$ обозначим сужение гамильтониана $H(\sigma)$ на $A$. Гамильтониан $H$ называется гамильтонианом ближайшего соседа, если

$$
H(\sigma)=\sum_{x \in \Lambda} H^{(1)}(\sigma(x))+\sum_{\substack{x, y \in \Lambda \\ c(x, y)=1}} H^{(2)}(\sigma(x), \sigma(y)) .
$$

ПрЕДЛОЖЕНИЕ 1. Пусть $\mu-p$-адическое гиббсовское состояние с гамильтонианом ближайшего соседа $H$. Тогда $\mu$-р-адическое марковское случайное поле.

ДоказатеЛьство. Пусть $A \cap B=\varnothing$ и $\partial A \subset B$. Тогда имеем

$$
\mu\left(\bar{\sigma}_{A} \mid \widetilde{\sigma}_{B}\right)=\frac{\sum_{\sigma_{\Lambda \backslash(A \cup B)}} \exp _{p}\left\{-H\left(\bar{\sigma}_{A}, \widetilde{\sigma}_{B}, \sigma_{\Lambda \backslash(A \cup B)}\right)\right\}}{\sum_{\sigma_{\Lambda \backslash(A \cup B), \bar{\sigma}_{A}}} \exp _{p}\left\{-H\left(\bar{\sigma}_{A}, \widetilde{\sigma}_{B}, \sigma_{\Lambda \backslash(A \cup B)}\right)\right\}},
$$

где $\sigma=\left(\bar{\sigma}_{A}, \widetilde{\sigma}_{B}, \sigma_{\Lambda \backslash(A \cup B)}\right)$. Далее,

$$
\begin{aligned}
H(\sigma)= & H_{A}\left(\bar{\sigma}_{A}\right)+H_{A, B}^{(2)}\left(\bar{\sigma}_{A}, \widetilde{\sigma}_{B}\right)+H_{B}\left(\widetilde{\sigma}_{B}\right)+ \\
& +H_{\Lambda \backslash(A \cup B)}\left(\sigma_{\Lambda \backslash(A \cup B)}\right)+H_{B, \Lambda \backslash(A \cup B)}^{(2)}\left(\widetilde{\sigma}_{B}, \sigma_{\Lambda \backslash(A \cup B)}\right),
\end{aligned}
$$

где энергия $H_{A, B}^{(2)}$ определяется следующим образом:

$$
H_{A, B}^{(2)}\left(\sigma_{A}, \sigma_{B}\right)=\sum_{\substack{x \in A, y \in B \\ c(x, y)=1}} H^{(2)}\left(\sigma_{A}(x), \sigma_{B}(y)\right) .
$$


Обозначим через $Z_{A}\left(\widetilde{\sigma}_{\partial A}\right)$ условную статистическую сумму:

$$
Z_{A}\left(\widetilde{\sigma}_{\partial A}\right)=\sum_{\bar{\sigma}_{A}} \exp _{p}\left\{-H_{A}\left(\bar{\sigma}_{A}\right)-H_{A, \partial A}^{(2)}\left(\bar{\sigma}_{A}, \widetilde{\sigma}_{\partial A}\right)\right\} .
$$

Отсюда числитель в правой части равенства (1) равен

$$
\exp _{p}\left\{-H_{A}\left(\bar{\sigma}_{A}\right)-H_{A, B}^{(2)}\left(\bar{\sigma}_{A}, \widetilde{\sigma}_{B}\right)-H_{B}\left(\widetilde{\sigma}_{B}\right)\right\} Z_{\Lambda \backslash(A \cup B)}\left(\widetilde{\sigma}_{B}\right),
$$

а знаменатель равен

$$
\exp _{p}\left\{-H_{B}\left(\widetilde{\sigma}_{B}\right)\right\} Z_{\Lambda \backslash(A \cup B)}\left(\widetilde{\sigma}_{B}\right) Z_{A}\left(\widetilde{\sigma}_{B}\right)
$$

Подставляя эти выражения в выражение (1) и заметив, что $H_{A, B}^{(2)}\left(\bar{\sigma}_{A}, \widetilde{\sigma}_{B}\right)=$ $H_{A, \partial A}^{(2)}\left(\bar{\sigma}_{A}, \widetilde{\sigma}_{\partial A}\right)$ и $Z_{A}\left(\widetilde{\sigma}_{B}\right)=Z_{A}\left(\widetilde{\sigma}_{\partial A}\right)$, мы после очевидных сокращений приходим к равенству

$$
\mu\left(\bar{\sigma}_{A} \mid \tilde{\sigma}_{B}\right)=\mu\left(\bar{\sigma}_{A} \mid \tilde{\sigma}_{\partial A}\right) .
$$

\section{4. ПРИМЕРЫ}

Как известно [2], в вещественном случае понятия меры Гиббса и марковского случайного поля совпадают. Но в поле $p$-адических чисел $\mathbb{Q}_{p}$, как это было доказано в работе [18], класс $p$-адических марковских случайных полей шире, чем класс $p$-адических мер Гиббса.

Приведем примеры $p$-адических марковских случайных полей, которые не являются $p$-адическими мерами Гиббса.

Пусть $\Lambda=\{x, y, z\}$ и $\Phi=\{0,1\}$. Как отмечалось выше, при $q=2$ множество конфигураций $\Omega$ можно отождествить с множеством всех подмножеств множества $\Lambda$.

ПримеР 1 . Пусть $c(x, y)=c(y, z)=c(x, z)=0$. В этом случае $p$-адическая вероятностная мера $\mu$ на $P(\Lambda)$ является марковским случайным полем тогда и только тогда, когда она удовлетворяет условиям

$$
\begin{aligned}
\mu(x, y) \mu(\varnothing) & =\mu(x) \mu(y), & \mu(x, z) \mu(\varnothing) & =\mu(x) \mu(z), \\
\mu(y, z) \mu(\varnothing) & =\mu(y) \mu(z), & \mu(x, y, z) \mu(\varnothing) & =\mu(x) \mu(y, z), \\
\mu(x, y, z) \mu(\varnothing) & =\mu(y) \mu(x, z), & \mu(x, y, z) \mu(\varnothing) & =\mu(z) \mu(x, y) .
\end{aligned}
$$

ПримеР 2. Пусть $c(x, y)=1, c(y, z)=c(x, z)=0$. В этом случае $p$-адическая вероятностная мера $\mu$ на $P(\Lambda)$ является марковским случайным полем тогда и только тогда, когда она удовлетворяет условиям

$$
\begin{array}{clrl}
\mu(x, z) \mu(\varnothing)= & \mu(x) \mu(z), & \mu(y, z) \mu(\varnothing) & =\mu(y) \mu(z), \\
\mu(x, y, z) \mu(\varnothing)= & \mu(z) \mu(x, y), & \mu(x, y, z) \mu(x) & =\mu(x, y) \mu(x, z), \\
& \mu(x, y, z) \mu(y)=\mu(x, y) \mu(y, z) .
\end{array}
$$

ПримеР 3. Пусть $c(x, y)=c(y, z)=1, c(x, z)=0$. В этом случае $p$-адическая вероятностная мера $\mu$ на $P(\Lambda)$ является марковским случайным полем тогда и только тогда, когда она удовлетворяет условиям

$$
\begin{gathered}
\mu(x, z) \mu(\varnothing)=\mu(x) \mu(z), \quad \mu(x, y, z) \mu(x)=\mu(x, y) \mu(x, z), \\
\mu(x, y, z) \mu(y)=\mu(x, y) \mu(y, z) .
\end{gathered}
$$


ПримеР 4. Пусть $c(x, y)=c(y, z)=c(x, z)=1$. В этом случае всякая $p$-адическая вероятностная мера $\mu$ на $P(\Lambda)$ является марковским случайным полем.

Найти общее решение каждой системы уравнений (3)-(5) трудно. Мы хотим найти хотя бы одно решение, не являющееся гиббсовским. Заметим, что множество таких решений имеет мощность континуума. Если $p$-адическую вероятностную меру $\mu$ на $P(\Lambda)$ определим следующим образом:

$$
\begin{gathered}
\mu(\varnothing)=p^{3}, \quad \mu(x, y)=\mu(x, z)=\mu(y, z)=p^{3}-2 p^{2}+p, \\
\mu(x)=\mu(y)=\mu(z)=p^{2}-p^{3}, \quad \mu(x, y, z)=-p^{3}+3 p^{2}-3 p+1,
\end{gathered}
$$

то она является решением систем (3)-(5).

Нетрудно показать, что мера (6) не является $p$-адическим гиббсовским состоянием с потенциалом ближайшего соседа. Действительно, $\mu$ может быть мерой Гиббса с потенциалом (гамильтонианом) $H$, определяемым по формуле

$$
H(A)=\log _{p}\left[\frac{\mu(A)}{\mu(\varnothing)}\right] \quad \forall A \subset \Lambda .
$$

Но для (6) мы имеем

$$
\left|\frac{\mu(x)}{\mu(\varnothing)}-1\right|_{p}=\left|\frac{p^{2}-p^{3}}{p^{3}}-1\right|_{p}=\left|\frac{1}{p}-2\right|_{p}=p>1 .
$$

Согласно лемме 1 из (8) следует, что гамильтониан (7) не определен для меры (6).

\section{5. СЛУЧАЙ СЧЕТНЫХ ГРАФОВ}

Пусть $\Gamma=(V, e)$ - счетный граф и $\Phi=\{1,2, \ldots, q\}$. Функции $\sigma: V \rightarrow \Phi$ называются конфигурациями. Обозначим через $\Omega=\Phi^{V}$ множество всех конфигураций на $V$. Мы будем рассматривать $\Omega$ как компактное хаусдорфово пространство, являющееся произведением $|V|$ экземпляров дискретного пространства $\{1,2, \ldots, q\}$. Обозначим множество конечных подмножеств из $V$ через $\mathcal{B}(V)$.

Если $A \subset V$, введем обозначение $\sigma_{[A]}=\left\{\varphi \in \Omega:\left.\varphi\right|_{A} \equiv \sigma_{A}\right\}$. Тогда $A \in \mathcal{B}(V), \sigma_{[A]}$ называется конечномерным цилиндром. Конечномерные цилиндры образуют базис открытых множеств топологии в $\Omega$.

Пусть $\mathcal{F}$ - алгебра, порожденная конечномерными цилиндрами. $p$-Адическая мера $\mu$ на $(\Omega, \mathcal{F})$ определяется как аддитивный ограниченный функционал:

1) $\mu\left(\sigma_{[A]} \cup \sigma_{[B]}\right)=\mu\left(\sigma_{[A]}\right)+\mu\left(\sigma_{[B]}\right)$, если $\sigma_{[A]} \cap \sigma_{[B]}=\varnothing$;

2) $\sup \left\{\left|\mu\left(\sigma_{[A]}\right)\right|_{p}: \sigma_{[A]} \in \mathcal{F}\right\}<\infty$.

Обозначим через $\mathcal{M}_{p}(\Omega)$ множество $p$-адических мер на $(\Omega, \mathcal{F})$, которое рассматривается как топологическое пространство со слабой топологией.

p-Адическая мера $\mu$ на $(\Omega, \mathcal{F})$ называется вероятностной мерой, если выполняется еще одно условие:

3) $\mu(\Omega)=1$.

Через $\mathcal{V}_{p}(\Omega)$ обозначим множество всех $p$-адических вероятностных мер на $\Omega$.

Пусть $A, B \in \mathcal{B}(V)$. Обозначим через

$$
\mu\left(\bar{\sigma}_{[A]} \mid \widetilde{\sigma}_{[B]}\right)=\frac{\mu\left(\bar{\sigma}_{[A]} \cap \widetilde{\sigma}_{[B]}\right)}{\mu\left(\widetilde{\sigma}_{[B]}\right)}
$$


условную вероятность того, что конфигурация $\sigma$ есть $\bar{\sigma}_{A}=\{\bar{\sigma}(x), x \in A\}$ на множестве $A$ при условии, что на множестве $B$ она равна $\widetilde{\sigma}_{B}=\{\widetilde{\sigma}(x), x \in B\}$.

ОПРЕДЕЛЕНИЕ 2. $p$-Адическая вероятностная мера $\mu \in \mathcal{V}_{p}(\Omega)$ называется $p$ - $a \partial u$ ческим марковским случайным полем, если:

1) $\mu\left(\sigma_{[A]}\right) \neq 0$ для всех конечномерных цилиндров $\sigma_{[A]}$;

2) при $A, B \in \mathcal{B}(V)$ справедливо равенство

$$
\mu\left(\bar{\sigma}_{[A]} \mid \widetilde{\sigma}_{[B]}\right)=\mu\left(\bar{\sigma}_{[A]} \mid \widetilde{\sigma}_{[\partial A \cap B]}\right) .
$$

Обозначим через $\mathcal{M} \mathcal{R} \mathcal{F}_{p}(\Omega)$ множество всех $p$-адических марковских случайных полей на $(\Omega, \mathcal{F})$.

Теорема 1. Множество $\mathcal{M R \mathcal { F }}_{p}(\Omega)$ является непустым замкнутым подмножеством в $\mathcal{M}_{p}(\Omega)$.

ДокАЗАтЕЛЬСтво. Покажем, что множество $\mathcal{M R \mathcal { F }}_{p}(\Omega)$ не пусто.

Пусть $V_{1} \subset V_{2} \subset \cdots \subset \bigcup_{n=1}^{\infty} V_{n}=V$, где $V_{n} \in \mathcal{B}(V), n=1,2, \ldots$.

Определим $p$-адическую вероятностную меру $\mu^{(n)}$ на $\Phi^{V_{n}}$ следующим образом:

$$
\mu^{(n)}\left(\sigma_{[A]}\right)=Z_{n}^{-1} a_{1}^{\nu_{\sigma_{A}}(1)} \times \cdots \times a_{q}^{\nu_{\sigma_{A}}(q)},
$$

где $a_{i} \in \mathbb{Q}_{p} \backslash\{0\}, \nu_{\sigma_{A}}(i)$ - число $i$ в конфигурации $\sigma_{A}, Z_{n}$ - нормирующая константа:

$$
Z_{n}=\sum_{\sigma_{[A]} \in \mathcal{F}_{n}} a_{1}^{\nu_{\sigma_{A}}(1)} \times \cdots \times a_{q}^{\nu_{\sigma_{A}}(q)} .
$$

Нетрудно видеть, что мера $\mu^{(n)}$, определенная формулой $(9)$, является $p$-адическим марковским случайным полем. Далее,

$$
\begin{gathered}
\sum_{\sigma_{\left[V_{n+1}\right]}} \mu^{(n+1)}\left(\bar{\sigma}_{\left[V_{n}\right]} \cap \sigma_{\left[V_{n+1}\right]}\right)=Z_{n+1}^{-1} \sum_{\sigma_{\left[V_{n+1}\right]}} \prod_{k=1}^{q} a_{k}^{\nu_{\bar{\sigma}_{\left[V_{n}\right]}}(k)+\nu_{\sigma_{\left[V_{n+1}\right]}}(k)}= \\
=\mu^{(n)}\left(\bar{\sigma}_{\left[V_{n}\right]}\right) \frac{\sum_{\sigma_{\left[V_{n+1}\right]}} \prod_{k=1}^{q} a_{k}^{\nu_{\sigma_{\left[V_{n+1}\right]}}(k)}}{\sum_{\sigma_{\left[V_{n+1}\right]}} \prod_{k=1}^{q} a_{k}^{\nu_{\sigma_{\left[V_{n+1}\right]}}(k)}}=\mu^{(n)}\left(\bar{\sigma}_{\left[V_{n}\right]}\right) .
\end{gathered}
$$

Следовательно, меры $\mu^{(n)}$ являются согласованными. Для таких мер согласно $p$-адическому аналогу теоремы Колмогорова (см. [12]) существует предельная мера $\mu$ на $(\Omega, \mathcal{F})$.

Для любых пар множеств $A, B \in \mathcal{B}(V)$ существует номер $n_{0} \in \mathbb{N}$ такой, что $A, B \subset V_{n}$ при $n \geqslant n_{0}$. Так как для всех $n \in \mathbb{N}$ имеет место $\left.\mu\right|_{V_{n}}=\mu^{(n)}$ и каждая мера $\mu^{(n)}$ является $p$-адическим марковским случайным полем, то для предельной меры $\mu$ выполняется равенство $\mu\left(\bar{\sigma}_{[A]} \mid \tilde{\sigma}_{[B]}\right)=\mu\left(\bar{\sigma}_{[A]} \mid \tilde{\sigma}_{[\partial A \cap B]}\right)$.

Теперь докажем, что множество $\mathcal{M} \mathcal{R} \mathcal{F}_{p}(\Omega)$ является замкнутым в пространстве $\mathcal{M}_{p}(\Omega)$ со слабой топологией. Пусть последовательность $\mu_{n} \in \mathcal{M R \mathcal { F }}_{p}(\Omega)$ сходится к некоторой мере $\mu \in \mathcal{M}_{p}(\Omega)$, т. е. $\mu_{n}\left(\sigma_{[A]}\right) \rightarrow \mu\left(\sigma_{[A]}\right)$ при $n \rightarrow \infty$ для любого конечномерного цилиндра $\sigma_{[A]}$. 
Далее, для любых $A, B \in \mathcal{B}(V)$ и $\sigma, \bar{\sigma} \in \Omega$ имеем

$$
\begin{aligned}
\mid \mu\left(\sigma_{[A]} \mid\right. & \left.\bar{\sigma}_{[B]}\right)-\left.\mu\left(\sigma_{[A]} \mid \bar{\sigma}_{[\partial A \cap B]}\right)\right|_{p}=\mid \mu\left(\sigma_{[A]} \mid \bar{\sigma}_{[B]}\right)-\mu_{n}\left(\sigma_{[A]} \mid \bar{\sigma}_{[B]}\right)+ \\
& +\mu_{n}\left(\sigma_{[A]} \mid \bar{\sigma}_{[B]}\right)-\mu_{n}\left(\sigma_{[A]} \mid \bar{\sigma}_{[\partial A \cap B]}\right)+ \\
& +\mu_{n}\left(\sigma_{[A]} \mid \bar{\sigma}_{[\partial A \cap B]}\right)-\left.\mu\left(\sigma_{[A]} \mid \bar{\sigma}_{[\partial A \cap B]}\right)\right|_{p} \leqslant \\
\leqslant & \max \left\{\left|\mu_{n}\left(\sigma_{[A]} \mid \bar{\sigma}_{[B]}\right)-\mu\left(\sigma_{[A]} \mid \bar{\sigma}_{[B]}\right)\right|_{p}, \mid \mu_{n}\left(\sigma_{[A]} \mid \bar{\sigma}_{[\partial A \cap B]}\right)-\right. \\
& \left.-\left.\mu\left(\sigma_{[A]} \mid \bar{\sigma}_{[\partial A \cap B]}\right)\right|_{p}\right\} .
\end{aligned}
$$

Так как $\mu_{n} \rightarrow \mu$ из из (10) следует, что $\mu\left(\sigma_{[A]} \mid \bar{\sigma}_{[B]}\right)=\mu\left(\sigma_{[A]} \mid \bar{\sigma}_{[\partial A \cap B]}\right)$. Это означает, что $\mu \in \mathcal{M R \mathcal { F }}_{p}(\Omega)$. Теорема доказана.

ЗАмечаниЕ 1 . Пусть $H: \Omega \rightarrow \mathbb{Q}_{p}$ - некоторый гамильтониан на $\Omega=\Phi^{V}$, удовлетворяющий условию $|H(\sigma)|_{p}<p^{-1 /(p-1)}$. Для двух конфигураций $\sigma, \eta \in \Omega$ определим

$$
(\sigma \eta)_{A}(x)= \begin{cases}\sigma(x), & x \in A, \\ \eta(x), & x \in V \backslash A .\end{cases}
$$

При фиксированном $\eta$ определим $p$-адическое гиббсовское состояние на цилиндре $\sigma_{[A]}:$

$$
\mu_{\eta}\left(\sigma_{[A]}\right)=Z_{A, \eta}^{-1} \exp _{p}\left\{-H\left((\sigma \eta)_{A}\right)\right\},
$$

где $Z_{A, \eta}$ - нормирующая константа, т. е.

$$
Z_{A, \eta}=\sum_{\sigma_{A}} \exp _{p}\left\{-H\left((\sigma \eta)_{A}\right)\right\}
$$

Однако если гамильтониан не удовлетворяет условию $|H(\sigma)|_{p}<p^{-1 /(p-1)}$, то равенство (11) не определено. Поэтому в $p$-адическом случае оказывается более эффективным понятие $p$-адического марковского случайного поля.

Благодарности. У.А. Розиков благодарен Centre de physique théorique (Mapсель, Франция) за финансовую поддержку пребывания в сентябре-декабре 2012 г., а также он благодарен CNRS за поддержку.

\section{Список литературы}

[1] Х.-О. Георги, Гиббсовские меры и фазовые переходь, Мир, М., 1992.

[2] C. J. Preston, Gibbs States on Countable Sets, Cambridge Tracts in Mathematics, 68, Cambridge Univ. Press, Cambridge, 1974.

[3] Я. Г. Синай, Теория фазовых переходов. Строгие результаты, Наука, М., 1980.

[4] A. Yu. Khrennikov, Non-Archimedean Analysis: Quantum Paradoxes, Dynamical Systems and Biological Models, Mathematics and its Applications, 427, Kluwer, Dordrecht, 1997.

[5] E. Marinary, G. Parisi, Phys. Lett. B, 203:1-2 (1988), 52-54.

[6] В. С. Владимиров, И. В. Волович, Е. И. Зеленов, p-Адический анализ и математическая физика, Наука, М., 1994.

[7] A. Yu. Khrennikov, S. Yamada, A. van Rooij, Ann. Math. Blaise Pascal, 6:1 (1999), 21-32.

[8] A. C. M. van Rooij, Non-Archimedean Functional Analysis, Monographs and Textbooks in Pure and Applied Mathematics, 51, M. Dekker, New York, 1978.

[9] S. Albeverio, W. Karwowski, Stochastic Process. Appl., 53:1 (1994), 1-22.

[10] S. Albeverio, X. Zhao, Ann. Probab., 28:4 (2000), 1680-1710. 
[11] K. Yasuda, Osaka J. Math., 37:4 (2000), 967-985.

[12] Н. Н. Ганиходжаев, Ф. М. Мухамедов, У. А. Розиков, Узб. матем. журн., 1998, № 4, 23-29.

[13] M. Khamraev, F. M. Mukhamedov, U. A. Rozikov, Lett. Math. Phys., 70:1 (2004), 17-28, arXiv: math-ph/0510021.

[14] A. Yu. Khrennikov, F. M. Mukhamedov, J.F.F. Mendes, Nonlinearity, 20:12 (2007), 2923-2937, arXiv: 0705.0244.

[15] F. M. Mukhamedov, U. A. Rozikov, Indag. Math. (N.S.), 15:1 (2004), 85-99.

[16] F. M. Mukhamedov, U. A. Rozikov, Infin. Dimens. Anal. Quantum Probab. Relat. Top., 8:2 (2005), 277-290.

[17] Н. Коблиц, р-Адические числа, р-адический анализ и дзета-функиии, М., Мир, 1982.

[18] У. А. Розиков, Ф.Ф. Тураев, Докл. АН РУз., 2011, № 2, 3-6.

Поступила в редакцию 16.10.2012 